\title{
The decreased expression of electron transfer flavoprotein $\beta$ is associated with tubular cell apoptosis in diabetic nephropathy
}

\author{
HUA WANG $^{1,2}$, HAOJUN ZHANG ${ }^{2}$, XIAOHONG CHEN $^{3}$, TINGTING ZHAO ${ }^{2}$, QIN KONG $^{1}$, MEIHUA YAN $^{2}$, \\ BINGXUAN ZHANG ${ }^{2}$, SIFAN SUN ${ }^{1}$, HUI-YAO LAN ${ }^{5}$, NING LI ${ }^{6}$ and PING LI ${ }^{1,2,4}$ \\ ${ }^{1}$ Graduate School of Peking Union Medical College, Chinese Academy of Medical Sciences \\ and Peking Union Medical College; ${ }^{2}$ Institute of Clinical Medical Sciences, China-Japan Friendship Hospital, \\ Beijing; ${ }^{3}$ Department of Nephrology, Chinese Medicine Hospital of Shaanxi, Xi'an, Shaanxi; ${ }^{4}$ Beijing Key Laboratory \\ for Immune-Mediated Inflammatory Diseases, Beijing; ${ }^{5} \mathrm{Li}$ Ka Shing Institute of Health Sciences and Department \\ of Medicine and Therapeutics, and Shenzhen Research Institute, Chinese University of Hong Kong, \\ Hong Kong SAR; ${ }^{6}$ Institute of Basic Medical Science, Peking Union Medical College, Beijing, P.R. China
}

Received August 13, 2015; Accepted March 8, 2016

DOI: $10.3892 /$ ijmm.2016.2533

\begin{abstract}
Tubular injury is closely correlated with the development of progressive diabetic nephropathy (DN), particularly in cases of type 2 diabetes. The apoptosis of tubular cells has been recognized as a major cause of tubular atrophy, followed by tubulointerstitial fibrosis. Electron transfer flavoprotein $\beta(\mathrm{ETF} \beta)$ is known as an important electron acceptor in energy metabolism, but its role in DN was not fully understood. In the present study, we examined the expression pattern of ETF $\beta$ using diabetic kidney samples and further investigated ETF $\beta$ involvement in tubular epithelial cell (TEC) apoptosis. Human renal biopsy specimens from patients with DN as well as a spontaneous rat model of diabetes using Otsuka LongEvans Tokushima fatty (OLETF) rats, were employed in order to examine the expression of ETF $\beta$ and cell apoptosis in kidneys during the development of DN (for the rats, at 36 and 56 weeks of age respectively). Moreover, ETF $\beta$ siRNA was used to investigate the role of ETF $\beta$ in the apoptosis of renal tubular cells. Our present results showed that the expression of ETF $\beta$ in the kidneys was progressively decreased both in patients with DN and OLETF rats, which coincided with progressive renal injury and TEC apoptosis. In addition, the in vitro study demonstrated that knockdown of ETF $\beta$ caused apoptosis in tubular cells, as proven by the increased expression of pro-apoptotic proteins and TUNEL assay. Therefore, the findings of our present study suggest that ETF $\beta$ plays an important role in renal tubular cell apoptosis during the progression of $\mathrm{DN}$.
\end{abstract}

Correspondence to: Professor Ping Li, Beijing Key Laboratory for Immune-Mediated Inflammatory Diseases, Institute of Clinical Medical Sciences, China-Japan Friendship Hospital, 2 East Yinghua Road, Chaoyang, Beijing 100029, P.R. China

E-mail:1p8675@163.com

Key words: electron transfer flavoprotein $\beta$, apoptosis, tubular injury, diabetic nephropathy

\section{Introduction}

Diabetic nephropathy (DN), as a common complication of diabetes mellitus, is the leading cause of end-stage renal disease and chronic renal failure (1). Although glomerular injury is thought to be the initiating factor of kidney damage in DN, previous research has indicated that tubular injury is also a key cause of chronic kidney injury (2), which correlates with the progression of DN (3). Tubular epithelial cell (TEC) apoptosis is considered a critical detrimental event which leads to kidney injury in cases of DN, and is closely associated with tubular atrophy and interstitial fibrosis (4). However, the mechanisms of tubular apoptosis during the progression of $\mathrm{DN}$ remain poorly understood.

Electron transfer flavoprotein $\beta(\mathrm{ETF} \beta)$ is a subunit of ETF that is comprised of $\alpha$ and $\beta$ subunits containing a flavin adenine dinucleotide (FAD) cofactor in the mitochondrial matrix. In the mitochondria, the oxidation of fatty acids is coupled with the mitochondrial transport chain through an electron transfer pathway, comprised of ETF-ubiquinone oxidoreductase (ETF-QO) and ubiquinone. Therefore, ETF acts as an electron acceptor of energy production from amino acid and fatty acids (5), which accepts electrons from flavoprotein dehydrogenases (6) and transfers them to ETF-QO in the inner mitochondrial membrane (7-9). Deficiency in human ETF results in a metabolic disease known as multiple acyl-CoA dehydrogenation deficiency (MADD) or glutaric acidemia type 2, and can even cause death in the neonatal period (10).

In the present study, in order to examine the role of ETF $\beta$ in DN, a spontaneous type 2 diabetic model using Otsuka Long-Evans Tokushima fatty (OLETF) rats was used in the present study. This rat model is characterized by obesity, insulin resistance, hyperglycemia, dyslipidemia and renal complications (11). More importantly, the renal abnormalities noted in OLETF rats resemble those of type 2 diabetic patients (11). Long-Evans Tokushima Otsuka (LETO) rats are the control counterpart animal model; these rats were developed from the same colony but do not develop diabetes. 
We previously observed a mutation of the ETF $\beta$ gene in the renal cortex of diabetic OLETF rats, which occured in conservative regions. The ETF $\beta$ gene mutation of H88L in OLETF rats suggested that ETF $\beta$ possibly plays a role in DN (unpublished data). Thus, in the present study, we examined the expression of ETF $\beta$ in DN patients and rats, and explored the mechanism of action in cell lines.

\section{Materials and methods}

Human kidney biopsy samples. Human kidney tissues were collected from patients in the Chinese Medicine Hospital of Shaanxi (Xi'an, China). The medical information of patients was obtained by review of medical records and the tissues for clinical diagnosis were obtained from archived kidney biopsy specimens or discarded nephrectomy samples. The kidney tissues were from patients diagnosed with DN or kidney carcinoma without diabetes as the control. All patients with DN were diagnosed based on the presence of diabetes, massive proteinuria, and other histological changes typical of DN. Patients were aged between 48 and 65 years, the female:male ratio was $1: 1$, and proteinuria ranged from 2.3 to $4.3 \mathrm{~g} / 24 \mathrm{~h}$. Normal tissues around the tumor from kidney carcinoma patients were employed as the normal control; these patients were aged 51-65 years, the female:male ratio was 1:2, and proteinuria $<0.2 \mathrm{~g} / 24 \mathrm{~h}$. The patients were matched in terms of age and gender information. Kidney tissues from the patients were fixed in $10 \%$ phosphate-buffered formalin solution, embedded in paraffin, and then sectioned to $3-\mu \mathrm{m}$ thickness and stained with periodic acid-Schiff (PAS), TUNEL or used for immunohistochemical analyses. The study was approved by the Research Ethics Board of the Chinese Medicine Hospital of Shaanxi, and all patients provided informed consent.

Animals. Male OLETF rats and corresponding controls, 4-week-old LETO rats, were kindly provided by the Tokushima Research Institute (Otsuka Pharmaceutical Co., Ltd., Tokushima, Japan). During the experiments, rats were housed at $22 \pm 3^{\circ} \mathrm{C}$ in an atmosphere with $50 \pm 10 \%$ humidity and a 12-h light/dark cycle. Animals had free access to standard rat chow and tap water. Seven rats from the LETO control group and seven rats from the OLETF group were sacrificed using chloral hydrate at 36 weeks of age. The remaining seven rats of each group were sacrificed at 56 weeks of age. After sacrifice, blood samples were collected from the abdominal aorta, and kidneys were removed and separated for histopathological examination and biological analysis. Paraffin sections from rats were made as described above. The severity of DN was assessed by measuring urinary protein levels and PAS staining of kidney tissues with subsequent histopathological analysis. Animal experiments were performed in accordance with the Guide for the Care and Use of Laboratory Animal of the National Institutes of Health. Procedures were approved by the Ethics Committee of Animal Experiments of the China-Japan Friendship Hospital (Beijing, China) (no. 08013).

Measuring body weight, blood glucose, blood lipids and urinary protein levels. The body weight of rats was measured at 4-week intervals. Blood was sampled from the tail vein also at 4-week intervals, and blood glucose was measured using a OneTouch Ultra II Blood Glucose monitoring system (LifeScan, Inc., Milpitas, CA, USA). Total cholesterol (CHO) and triglyceride (TG) concentrations were measured using an AutoAnalyzer (Abbott Diagnostics, Abbott Park, IL, USA). Rats were housed in individual metabolic cages (Fengshi Laboratory Animal Equipment Co., Ltd., Suzhou, Jiangsu, China) for $24 \mathrm{~h}$ urine collection at 4-week intervals. Urinary protein was assessed using the Bradford method.

Kidney histological and immunohistochemical analyses. In the present study, PAS staining was performed on paraffinembedded tissues from patients and rats using standard protocols. The degree of glomerulosclerosis, which was defined as a thickening of the basement membrane and mesangial expansion, was evaluated in 40 counted glomeruli using a microscope at x400 magnification (BX51; Olympus, Tokyo, Japan), as previously described (12). The observer was blinded to all tissue samples. Tubulointerstitial injury was assessed in PAS-stained paraffin sections from rats at x200 magnification using a similar scoring system (grades 1-6) as has been previously described (12). Briefly, 10 random cortical fields under x200 magnification were outlined and positive staining patterns were subsequently identified. The percentage of positive area in the examined field was then measured, as previously described (13). Data are expressed as the means \pm SE. All counting was performed on blinded slides.

Immunohistochemical analysis was performed as described in our previous study (12). Briefly, rat kidneys from each group were fixed in $10 \%$ formalin, embedded in paraffin, and cut into $3-\mu \mathrm{m}$ thick sections followed by rehydration. Antigen retrieval was undertaken in $10 \mathrm{mM}$ citrate buffer $(\mathrm{pH}$ 6.0) at $90-95^{\circ} \mathrm{C}$ for $25 \mathrm{~min}$. Kidney sections were incubated with polyclonal goat ETF $\beta$ antibody (1:200; sc-242638) (Santa Cruz Biotechnology, Inc., Dallas, TX, USA), overnight at $4^{\circ} \mathrm{C}$. Following washing, slides were incubated with anti-rabbit secondary antibody in the same buffer. Immunohistochemical staining was performed using the GT Vision III Detection System/Mo Rb (Gene Tech Biotechnology Co., Ltd., Shanghai, China) and captured using a light microscope (Olympus) and quantified using Image Pro-Plus 6.0 software (Media Cybernetics, Inc., Rockville, MD, USA).

Cell culture and RNA interference. The normal rat kidney epithelial cell line (NRK-52E) was purchased from the Institute of Biochemistry and Cell Biology (Shanghai, China) and cultured in Dulbecco's modified Eagle's medium (DMEM; HyClone, Logan, UT, USA) supplemented with $25 \mathrm{mM}$ D-glucose, $5 \%$ fetal bovine serum (FBS; Gibco Life Technologies, Carlsbad, CA, USA) in an incubator with $5 \% \mathrm{CO}_{2}$ at $37^{\circ} \mathrm{C}$. Cells were cultured until they reached $70 \%$ confluence, and this was followed by transfection with siRNA for $48 \mathrm{~h}$.

NRK-52E cells were transfected with siRNA $(20 \mu \mathrm{m})$ targeting ETF $\beta$ or scrambled siRNA as negative control using Lipofectamine 3000 reagent (Invitrogen Life Technologies, Carlsbad,CA,USA) according to the manufacturer's instructions. The siRNA sequences were as follows: sense, 5'-GGU GACUGCUGACUUAAGATT-3' and antisense, 5'-UCUUAA GUCAGCAGUCACCTT-3' (purchased from GenePharma, Shanghai, China). The scrambled siRNA sequences were as follows: sense, 5'-UUCUCCGAACGUGUCACGUTT-3' and 
A Normal control

DN
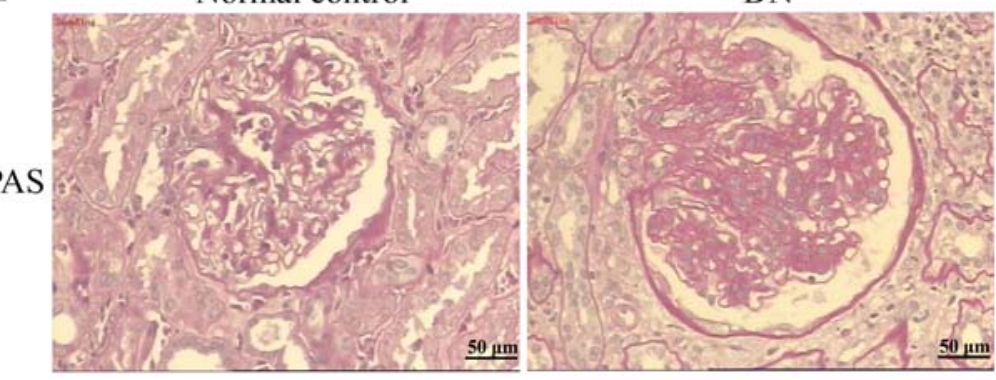

B

Normal control

$\mathrm{DN}$

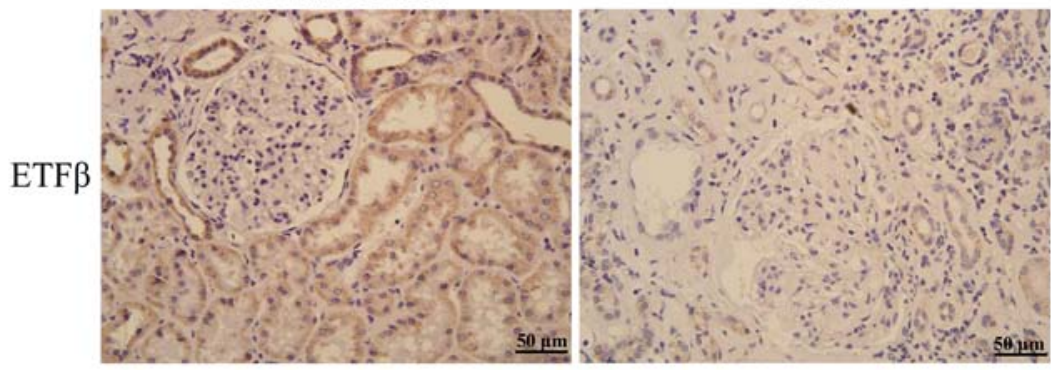

$\mathrm{C}$

Normal control

$\mathrm{DN}$

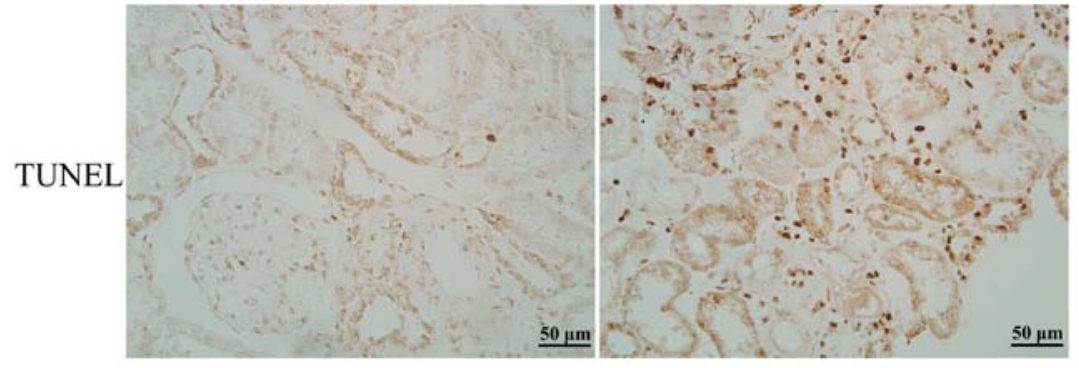

Figure 1. Expression of electron transfer flavoprotein $\beta(E T F \beta)$ and apoptosis detection in kidney samples of patients with diabetic nephropathy (DN) and normal tissues from around the kidney tumor. (A) Periodic acid-Schiff (PAS) staining (original magnification, x400) in human kidney tissues. (B) Expression of ETF $\beta$ in human biopsy samples, detected by immunohistochemical analysis. (C) Apoptosis in cells from human kidney cortex biopsy specimens, as detected by TUNEL assay. These stainings are from ten patients with DN and three patients with kidney carcinoma, respectively.

antisense, 5'-ACGUGACACGUUCGGAGAATT-3'. After transfection for $48 \mathrm{~h}$, cells were harvested for total protein extraction or TUNEL detection. Untreated cells with no transfection were used as the nomal group.

TUNEL assay. For in vivo detection of apoptosis, apoptotic cells in the human and rat kidneys were examined by TUNEL assay according to the manufacturer's instructions (Roche Diagnostics GmbH, Mannheim, Germany). After formalin-fixed sections were dewaxed, the slides were heated with a microwave for $1 \mathrm{~min}$ in $0.1 \mathrm{M}$ citrate buffer ( $\mathrm{pH} 6.0$ ), and rapidly cooled by adding doubled-distilled water and then transferred to PBS. The slides were blocked with buffer comprising $0.1 \mathrm{M}$ Tris-Hcl, 3\% BSA and 20\% bovine serum (pH 7.5) for $30 \mathrm{~min}$ at room temperature. DNA fragments were then labeled by terminal transferase dUTP for $30 \mathrm{~min}$ at $37^{\circ} \mathrm{C}$. In order to quantify apoptosis, the positively stained cells were calculated in 10 randomly selected fields in each slide as previously described (14).

For in vitro detection of apoptosis, NRK-52E cells were seeded in 96-well plates and transfected with ETF $\beta$ siRNA for $48 \mathrm{~h}$ as mentioned above. The cells were then rinsed twice, fixed in 4\% paraformaldehyde for $20 \mathrm{~min}$, and subsequently permeabilized with $0.1 \%$ Triton X-100 in PBS/BSA solution. TUNEL assay was performed according to the manufacturer's instructions and quantified as above.

Reverse transcription-quantitative polymerase chain reaction (RT-qPCR). Total RNA was extracted from rat kidney cortices with TRIzol reagent (Invitrogen Life Technologies). cDNA was synthesized using a RevertAid First Strand cDNA Synthesis kit (Thermo Fisher Scientific, Waltham, MA, USA), and RT-qPCR was performed using UltraSYBR Mixture (CWBio, Inc., Beijing, China). Amplification was performed as follows: initial denaturation at $95^{\circ} \mathrm{C}$ for $2 \mathrm{~min}$, followed by 40 cycles of denaturation at $95^{\circ} \mathrm{C}$ for $30 \mathrm{sec}$, annealing at $60^{\circ} \mathrm{C}$ for $30 \mathrm{sec}$ and final extension at $72^{\circ} \mathrm{C}$ for $2 \mathrm{~min}$, using an ABI 7500 thermocycler (Applied Biosystems, Foster, CA, USA). The primers used to detect ETF $\beta$ were: forward, 5'-GTACATTCGCCTCTCAG GTG-3' and reverse, 5'-TAC TCCTGCTAAGCGCTGAG- 3 '. The $\beta$-actin primers were forward, 5'-GACATCCGTAAAG ACCTCTATGCC-3' and reverse, 5'-ATAGAGCCACCAATC CACACAGAG-3'. Results were normalized to $\beta$-actin expression using the $\Delta \Delta C(t)$ method. 

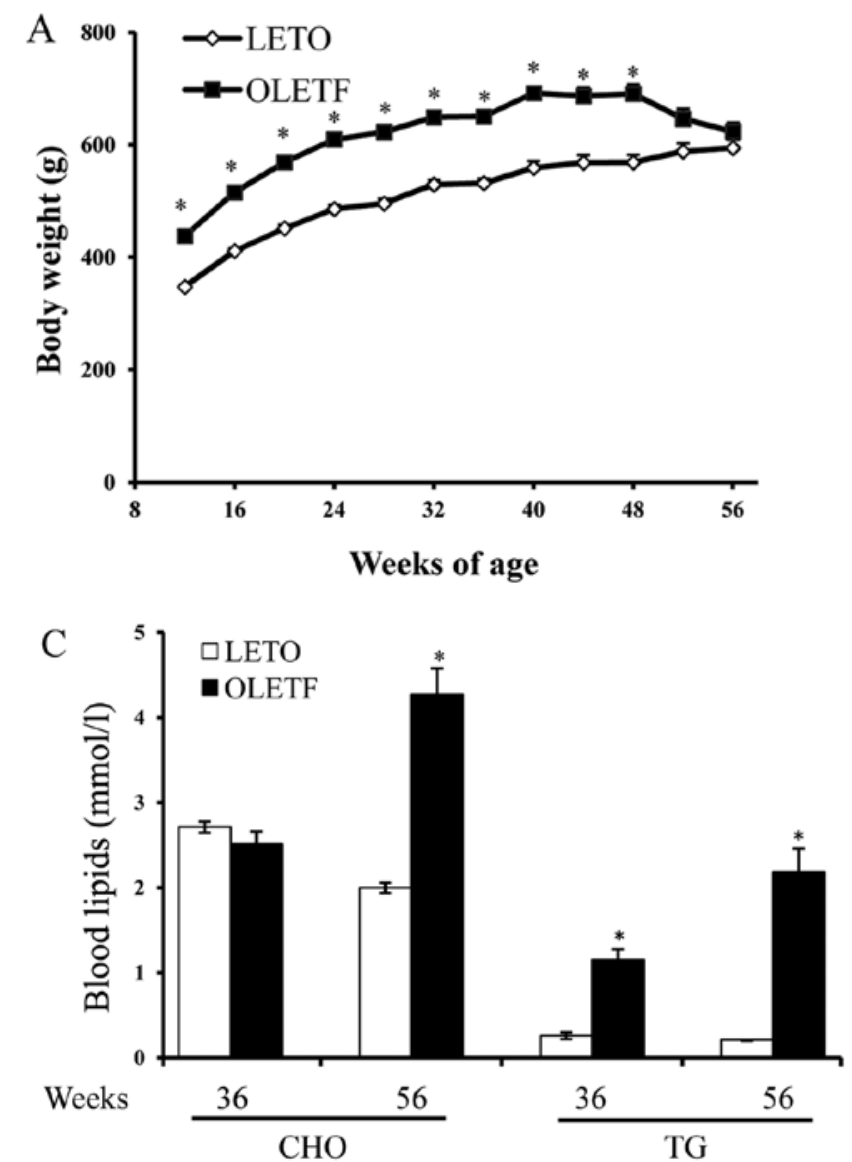
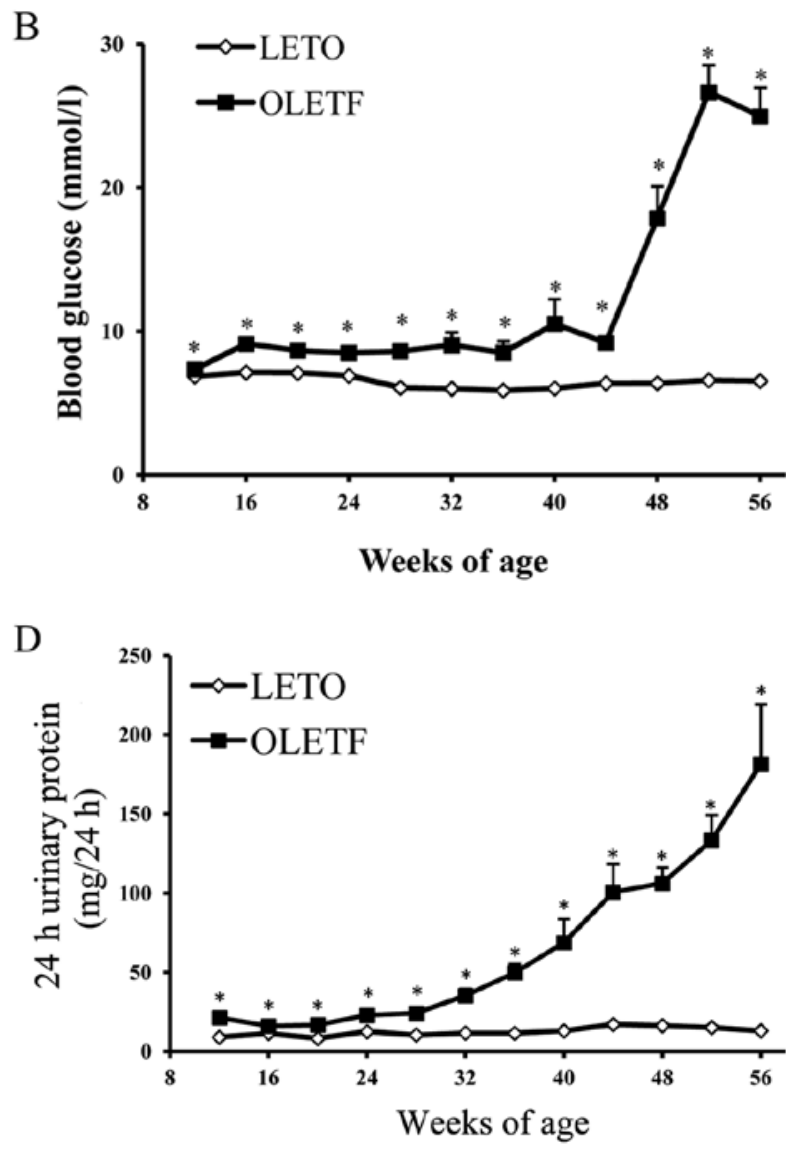

Figure 2. (A) Body weight, (B) blood glucose, (C) blood lipids and (D) 24 h urinary protein levels of Otsuka Long-Evans Tokushima fatty (OLETF) and LongEvans Tokushima Otsuka (LETO) rats during progression to severe diabetic nephropathy (DN). Data are expressed as the means \pm SEM of 7 rats from each group. ${ }^{*} \mathrm{P}<0.01$ compared with LETO rats at the corresponding intervals.

Western blot analysis. In the present study, proteins for western blot analysis were extracted from the rat kidney tissues or NRK-52E cells by lysing with radioimmunoprecipitation assay (RIPA) buffer, as previously described (12). Mitochondrial proteins from rat kidneys were extracted and separated from cytosolic proteins as previously described (15). Briefly, the tissues were homogenized in $0.25 \mathrm{M}$ sucrose and $10 \mathrm{mM}$ Hepes, $\mathrm{pH}$ 7.0, in a glass homogenizer equipped with a motor-driven Teflon Pestle (1,000 rpm, five up-and-down). Mitochondria were prepared by centrifugation for $70 \mathrm{~min}$ at $70,000 \times \mathrm{g}$ twice. Mitochondria were first collected at the interface $0.25 \mathrm{M} / 1.6 \mathrm{M}$ sucrose and then recovered under $1.45 \mathrm{M}$ sucrose. As final purification steps, mitochondria pellets were submitted to successive washes at $23,000 \mathrm{x} \mathrm{g}$, for $10 \mathrm{~min}$ and stored in liquid nitrogen. Protein concentration was tested by Bradford assay (Bio-Rad Laboratories, Inc., Hercules, CA, USA). Protein extracts (30-40 $\mu \mathrm{g}$ ) were loaded on $12 \%$ SDS-PAGE and analyzed by western blot analysis. After blocking with $5 \%$ skim milk, membranes were then incubated with the following primary antibodies: ETF $\beta$ goat antibody at a 1:1,000 dilution (sc-242638), BAX mouse antibody at a dilution of 1:1,000 (sc-7480) and Bcl-2 mouse antibody at a dilution of 1:1,000 (sc-7382) (all purchased from Santa Cruz Biotechnology, Inc.) and cleaved caspase-3 rabbit antibody at a dilution of 1:500 (SAB 4503294) (Sigma-Aldrich, St. Louis, MO, USA). $\beta$-actin mouse antibody was used as the loading control for total protein at a dilution of 1:3,000 (KM 9001)
(Tianjin Sungene Biotech Co., Ltd., Tianjin, China), and VDAC rabbit antibody (\#4866) (Cell Signaling Technology, Danvers, MA, USA) was used as the loading control for mitochondrial protein at a dilution of 1:1,000, overnight. Bands were detected using an ECL Western Blot Detection kit (Amersham Pharmacia Biotech, San Francisco, CA, USA) and subsequently quantified by densitometry using Quantity One software.

Statistical analyses. All values are expressed as the means \pm SEM. Results were analyzed using ANOVA or two-tailed Student's t-test. A P-value $<0.05$ was considered to indicate a statistically significant difference.

\section{Results}

Decreased expression of ETF $\beta$ in kidneys of patients with DN is accompanied by excessive numbers of apoptotic cells. Peri-carcinoma tissues from patients were used as a control, and kidney tissues from patients with DN were enrolled in the study; ten patients with DN and three patients with kidney carcinoma were enrolled in the study. Biopsies confirmed the presence of the disease, and PAS staining provided evidence for these pathological features (Fig. 1A). We examined expression patterns of ETF $\beta$ protein in the kidney cortices of non-diabetic and diabetic humans. Compared with the normal tissue around the tumors, the expression of ETF $\beta$ was greatly reduced in 
A

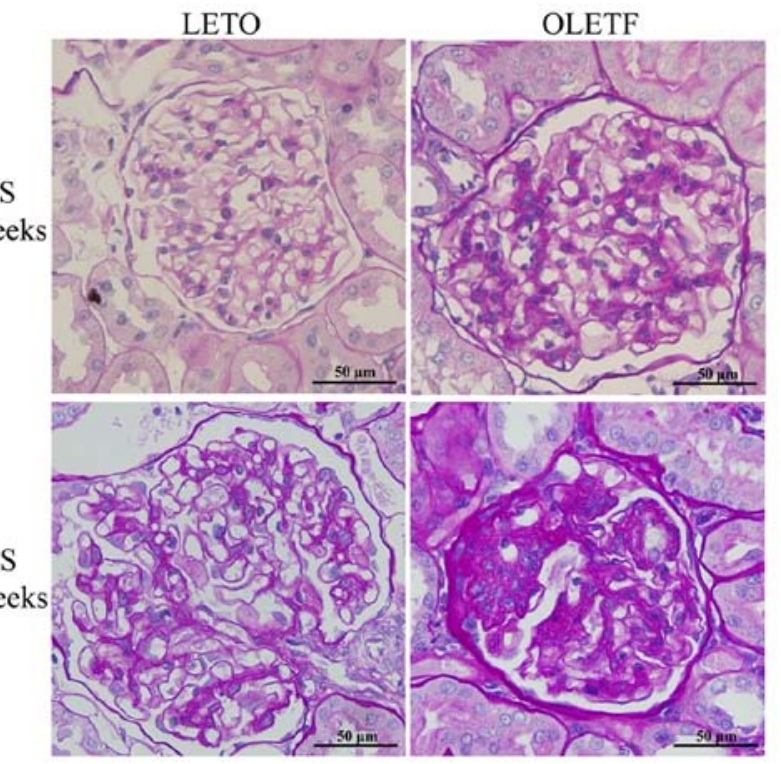

$\mathrm{C}$

C
PAS
36 weeks

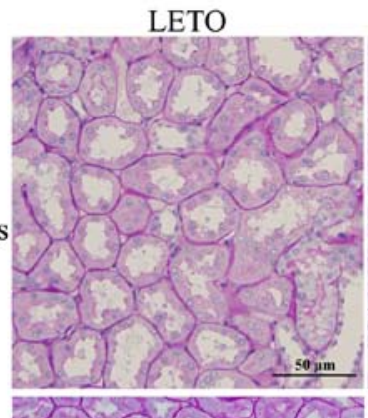

OLETF

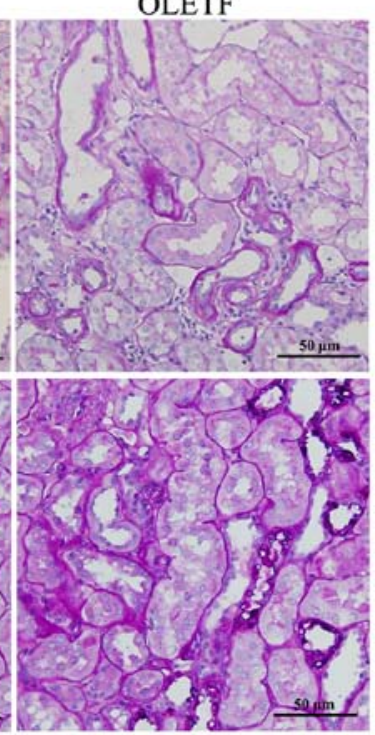

$\mathrm{B}$

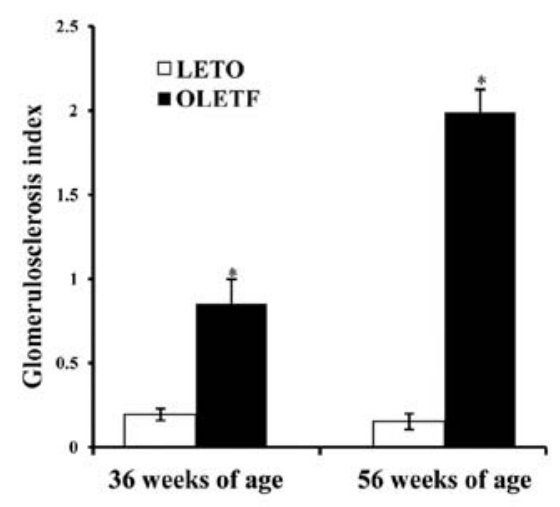

$\mathrm{D}$

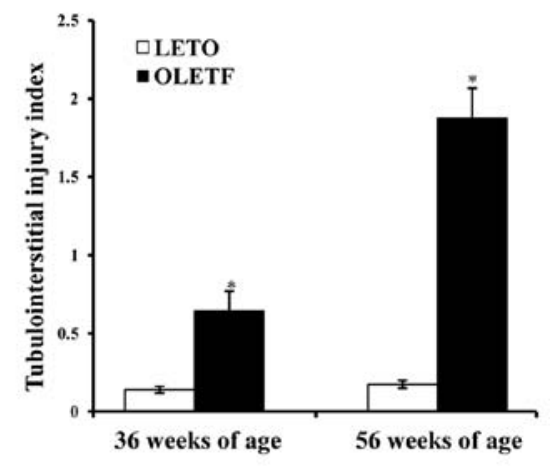

Figure 3. Periodic acid-Schiff (PAS) staining (original magnification, $\mathrm{x} 400$ ) of the renal glomerular and tubular regions of Otsuka Long-Evans Tokushima fatty (OLETF) and Long-Evans Tokushima Otsuka (LETO) rats at 36 and 56 weeks of age respectively. (A) PAS staining of renal glomerulus and (B) semiquantitative analysis of glomerulosclerosis. (C) PAS staining of renal tubules and (D) semiquantitative analysis of tubulointerstitial injury. Data are expressed as the means \pm SEM from 7 rats. ${ }^{*} \mathrm{P}<0.01$ compared with LETO rats.

kidney biopsy samples from patients with DN (Fig. 1B). In addition, we noted that the distribution of ETF $\beta$ was primarily in the TECs, but rarely in the glomeruli. Excessive apoptotic nuclei were observed in the kidney tissues of DN patients using a TUNEL assay (Fig. 1C). These findings are suggestive of a possible association between decreased tubular ETF $\beta$ expression and TEC apoptosis in cases of human DN.

Progression of DN in OLETF rats. In the present study, the body weight of OLETF rats was significantly greater than that of LETO rats from 12 to 48 weeks (Fig. 2A). However, as diabetes developed, no significant difference was observed between 53 and 56 weeks. The blood glucose levels of OLETF rats were significantly elevated at 48 weeks and peaked at 53 weeks (Fig. 2B), which was associated with the development of proteinuria (Fig. 2D), glomerulosclerosis index and tubulointerstitial injury index (Fig. 3). Moreover, high levels of blood lipids, including serum $\mathrm{CHO}$ and TG concentration were marked at 56 weeks of age (Fig. 2C). Serum TG level increased at an early stage, whereas serum CHO levels were elevated later. There was mild glomerular and tubular injury as early as 36 weeks of age, and this then progressed to severe glomerulosclerosis and tubular atrophy and tubulointerstitial fibrosis at 56 weeks (Fig. 3).

In the kidney cortices of OLETF rats, elevated expression of BAX/Bcl-2 and cleaved caspase-3 occurred only at 56 weeks of age (Fig. 4A and B), whereas there was no significant change in expression at 36 weeks of age $(\mathrm{P}>0.05$, data not shown). In agreement with the molecular changes involved in apoptosis, the occurrence of apoptosis in the diabetic kidneys also increased in 56-week-old OLETF rats, as evidenced by the results of the TUNEL assay (Fig. 4C and D). 
A

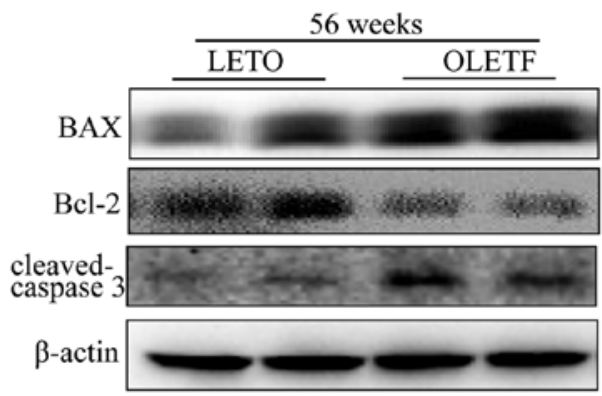

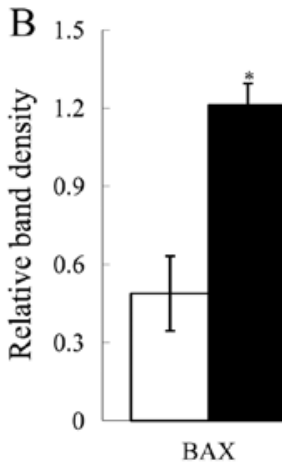

口LETO

- OLETF

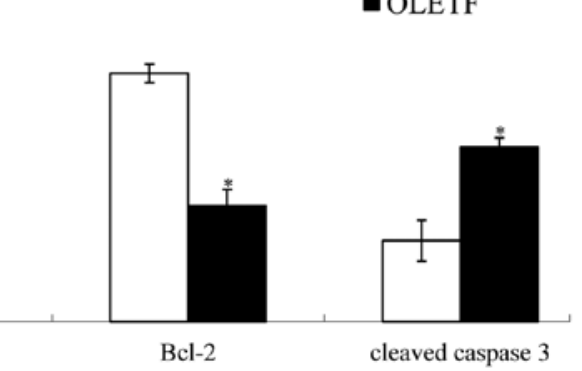

$\mathrm{C}$

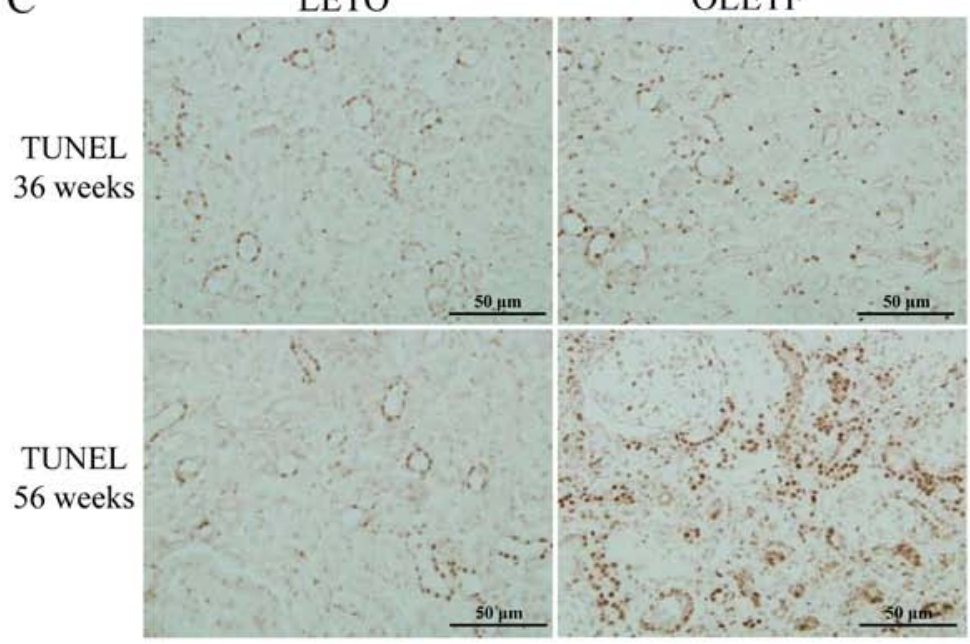

$\mathrm{D}$

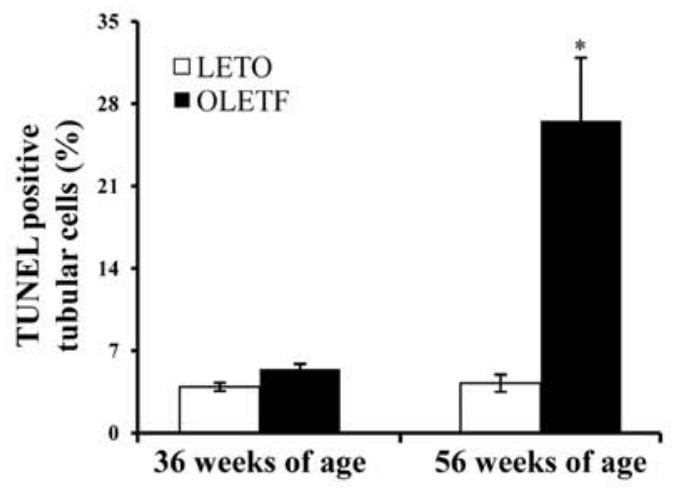

Figure 4. Apoptosis-related protein expression in 56-week-old rats and TUNEL staining using kidney samples from Otsuka Long-Evans Tokushima fatty (OLETF) and Long-Evans Tokushima Otsuka (LETO) rats at 36 and 56 weeks of age. (A) Representative western blots of BAX, Bcl-2, and cleaved caspase-3 in the kidney cortices of OLETF and LETO rats at 56 weeks of age. (B) Histogram shows semiquantitative analysis for each band. (C) Apoptotic cells in the kidney cortex, as shown by TUNEL positive staining (original magnification, $\mathrm{x} 400$ ) in rats at the two time points. (D) Histogram shows semiquantitative analysis of tubular cell apoptosis. Data are expressed as the means \pm SEM from 7 rats. ${ }^{*} \mathrm{P}<0.01$ compared with LETO rats.

Decreased expression of ETF $\beta$ in diabetic kidney and the progression of DN in OLETF rats. By contrast, we noted that the expression of ETF $\beta$ was high in the kidneys of control LETO rats, but it was markedly decreased in the kidneys of diabetic OLETF rats (Fig. 5). ETF $\beta$ mRNA and protein levels were both markedly downregulated only at 56 weeks of age (Fig. 5A and B). ETF $\beta$ expression was also reduced in mitochondria, which confirmed the ETF $\beta$ decrease prior to loss of mitochondria, since we separated the mitochondrial fraction and tested ETF $\beta$ expression with VDAC as a mitochondria control (Fig. 5C). Immunohistochemistry results indicated that ETF $\beta$ was widely expressed and located in normal tubular region, compared with glomerular region (Fig. 5D and E).

In 56-week-old OLETF rats we noted increased levels of $\mathrm{BAX} / \mathrm{Bcl}-2$ ratio and cleaved caspase 3, and decreased ETF $\beta$ in kidneys, which was linked with TEC apoptosis, as demonstrated by the significant increase in TUNEL-positive cells (Fig. 4).

Knockdown of ETF $\beta$ results in NRK-52E cell apoptosis. In order to better understand the functional role of ETF $\beta$ in TEC apoptosis, we knocked down ETF $\beta$ in NRK-52E cells. As shown in Fig. 6A and B, knockdown of ETF $\beta$ reduced the protein expression levels of ETF $\beta$ significantly, which elevated expressions of BAX and cleaved caspase-3. There was also decreased expression of $\mathrm{Bcl}-2$. Moreover, in ETF $\beta$ siRNA treated cells, inhibiton of ETF $\beta$ expression caused increased apoptosis of cells compared with normal cells or cells transfected with scrambled siRNA (Fig. 6C).

\section{Discussion}

Although tubular atrophy and tubulointerstitial fibrosis associated with tubular apoptosis are the most convincing evidence for the progression of DN (16), the mechanisms underlying this progression were poorly understood. In the present study, we noted excessive apoptosis, which was associated with progressive renal injury in diabetic patients and rats. To our surprise, we discovered that a significant decrease in ETF $\beta$ was associated with an increase in TEC apoptosis and progressive renal injury in the diabetic patients and rats, which suggests a negative link between ETF $\beta$ expression and TEC apoptosis. Importantly, downregulation of ETF $\beta$ by employing siRNA resulted in significant apoptosis of NRK-52E cells. Therefore, we suggest that decreased expression of ETF $\beta$ is closely related to apoptosis in TECs.

Tubular cell apoptosis occurs earlier than the onset of kidney fibrosis, and results in matrix accumulation in the tubulointerstitium (17). Previous research has shown that apoptosis is one of the pathological features of progressive kidney injury in patients with diabetes (18). Oxidative 

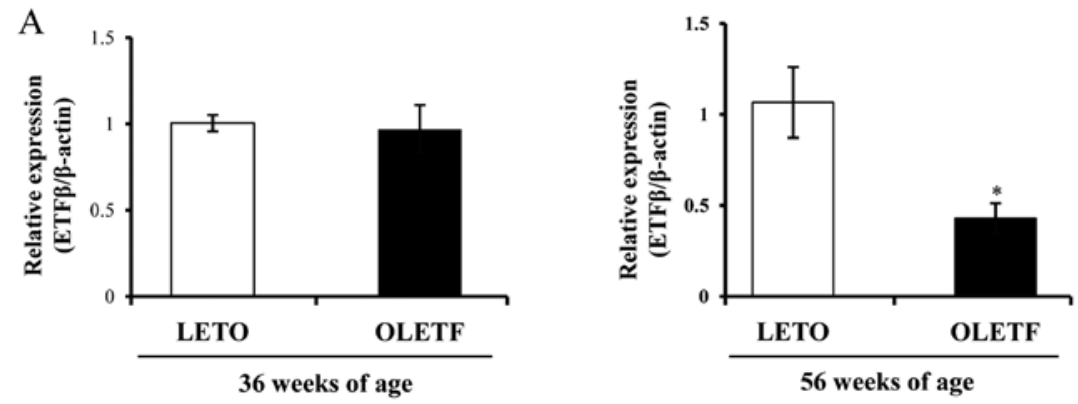

B Total protein
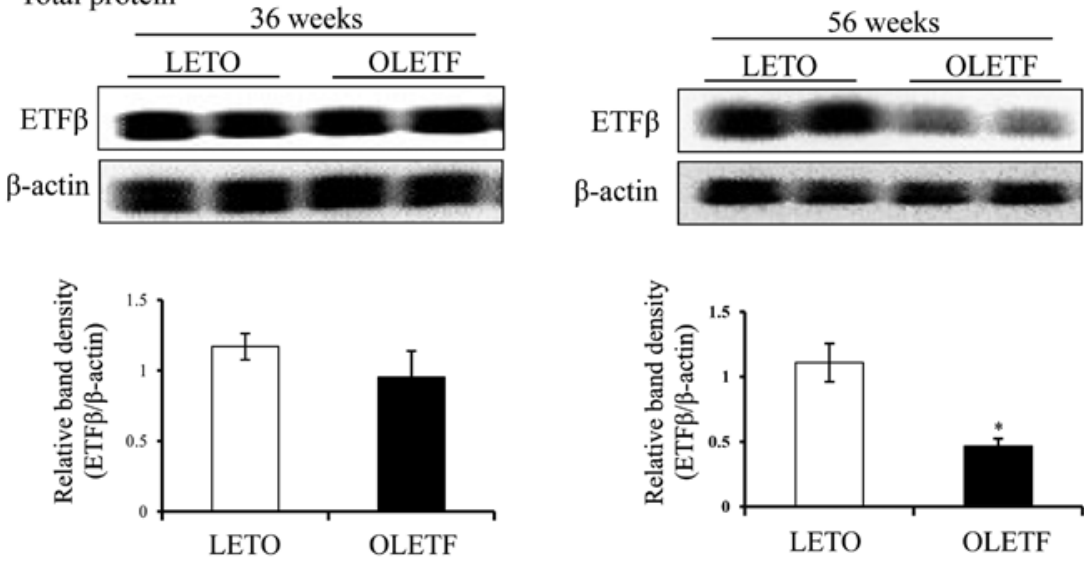

C Mitochondrial fraction
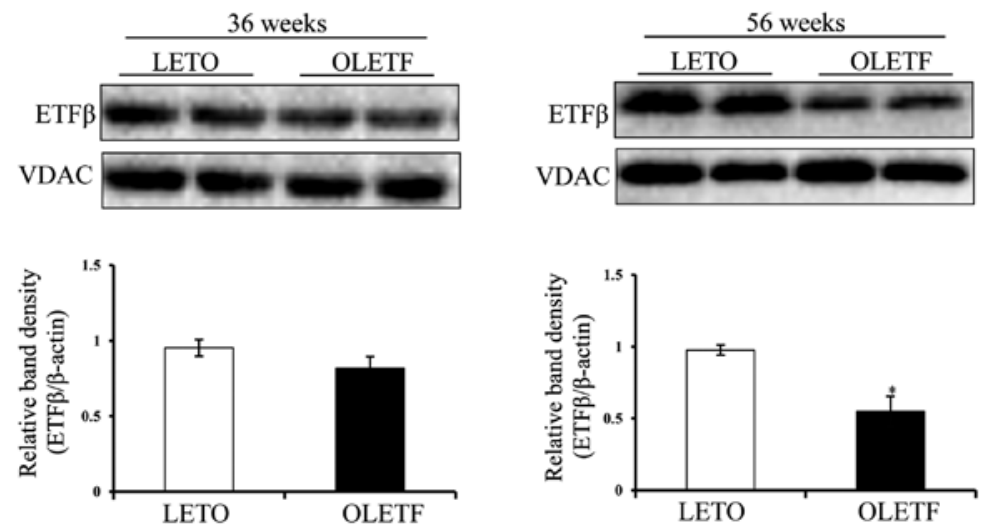

$\mathrm{D}$

36

weeks

56

weeks
LETO

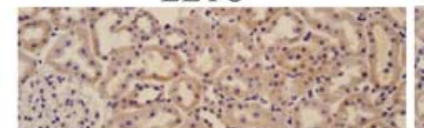

OLETF

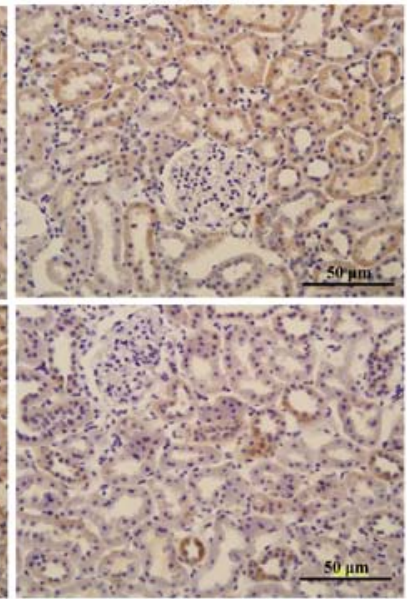

E

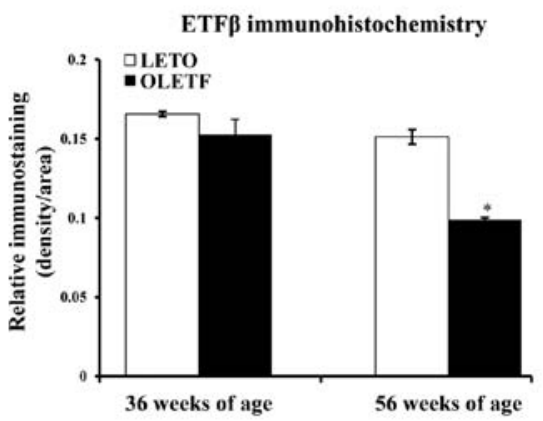

Figure 5. Expression of electron transfer flavoprotein $\beta$ (ETF $\beta$ ) in the kidneys during the progression of diabetic nephropathy (DN) in rats at 36 and 56 weeks of age respectively. (A) mRNA levels of ETFß in the kidneys of Otsuka Long-Evans Tokushima fatty (OLETF) vs. Long-Evans Tokushima Otsuka (LETO) rats, as measured by RT-qPCR. (B) The expression of ETF $\beta$ in the kidney cortices of rats, as measured by western blot analysis. (C) ETF $\beta$ protein levels in the mitochondrial fraction from the kidney cortices of rats, as measured by western blot analysis. (D) Representative pictures of immunostaining (original magnification, $\mathrm{x} 400$ ) of ETF $\beta$ and (E) semiquantitative analysis. Data are expressed as the means \pm SEM from 7 rats. ${ }^{*}$ P $<0.01$ compared with LETO rats. 
A

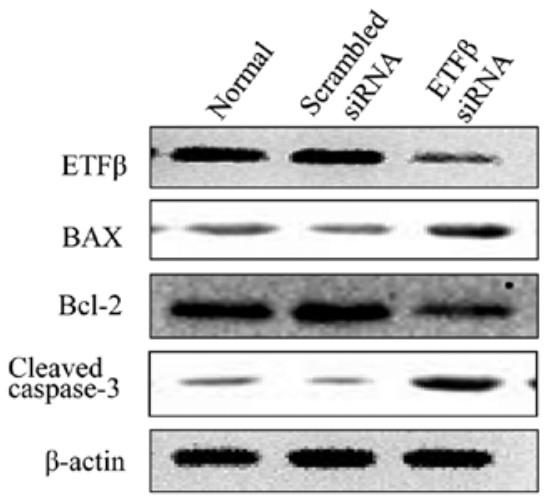

$\mathrm{C}$

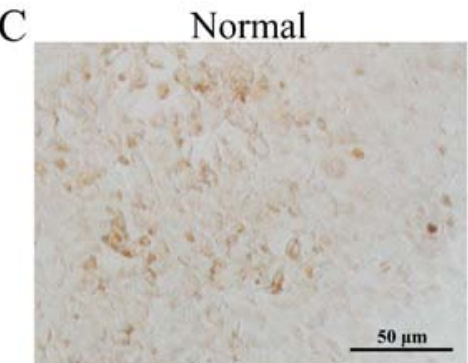

ETF $\beta$ siRNA

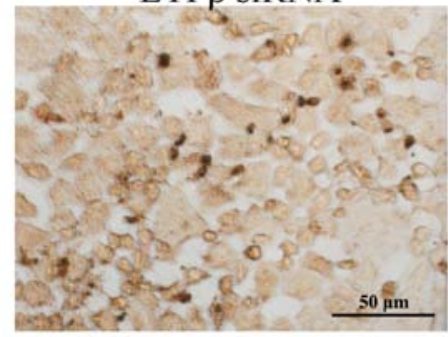

B

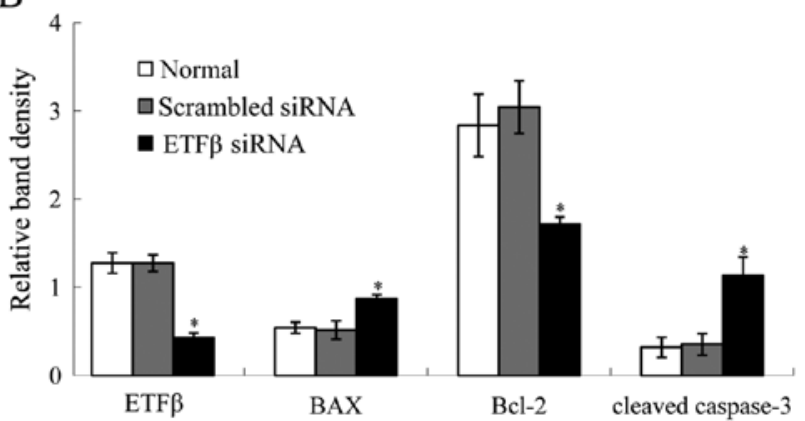

Figure 6. Effect of electron transfer flavoprotein $\beta$ (ETF $\beta$ ) knockdown on apoptosis in normal rat kidney epithelial (NRK-52E) cells. (A) Effect of ETF $\beta$ knockdown on the expression of apoptosis-related proteins. (B) Histogram represents the semiquantitative analysis of bands. (C) Apoptotic ratio of NRK-52E cells (original magnification, $\mathrm{x} 400$ ) as evidenced by TUNEL staining and histogram showing the semiquantitative analysis results. Data are expressed as the means \pm SEM from three independent experiments. ${ }^{*} \mathrm{P}<0.01$ compared with scrambled siRNA group.

stress and mitochondrial dysfunction have been reported to contribute to tubular cell apoptosis $(19,20)$, but the mechanisms mediating excessive apoptosis during the course of DN have not been fully explored. Downregulation of ETF $\beta$ associated with type 2 diabetes was noted using a quantitative proteomic approach and mouse pancreatic islets (21). In the present study, we discovered that decreased ETF $\beta$ resulted in tubular cell apoptosis, which is a cause of DN.

In the present study, we found that decreased ETF $\beta$ expression was accompied by tubular cell apoptosis both in human samples and rat kidney samples. The elevated BAX/Bcl-2 ratio in diabetic OLETF rats activates subsequent apoptotic signaling and cleaves pro-caspase-3 to the active form (22). Cleaved caspase-3 expression is considered a hallmark of apoptosis (23), and our data demonstrated eleveated cleaved caspase-3 expression in the kidney cortices of 56-week-old diabetic rats, which was consistent with decreased expression of ETF $\beta$ and increased TUNEL-positive stainings.

The suppression of ETF $\beta$ in TECs may be a result of the diabetic conditions of high glucose and dyslipidemia; in fatty acid $\beta$-oxidation, amino acid oxidation, and choline metabolism, ETF is positioned at a key metabolic branch point, as it accepts electrons from at least nine dehydrogenases and transfers them to the membrane-bound respiratory chain (24). According to functional and structural research of ETF $\beta$, the absence of the ETF $\beta$ subunit is a result of its role as a 'fixed' electron carrier, but it should be flexible to adapt different structural dehydrogenases upstream (8). However, the mechanism by which ETF $\beta$ is regulated in cases of diabetes is still unclear and requires further investigation. Previous study has demonstrated that elevation of upstream dehydrogenase by peroxisome proliferator-activated receptor $\delta$ attenuated apoptosis induced by fatty acids in a $\beta$-cell line (25). Moreover, the expression of ETF is significantly downregulated in human vein endothelial cells when apoptosis is induced by digoxin (26).

The results of our present study demonstrated that decreased expression of ETF $\beta$ was associated with TEC apoptosis, suggesting that ETF $\beta$ plays a protective role in TEC apoptosis. In the tubular regions, the mitochondrial $\beta$-oxidation pathway is much better developed than in glomerular regions (27). Therefore, as the electron receptor for the first reaction of fatty acid $\beta$-oxidation, ETF $\beta$ deficiency in the tubular cells may cause decreased energy supply (28). Also, since the physiological upstream acceptors for the electrons are missing, decreased expression of ETF $\beta$ may result in generation of excessive ROS (29). Oxidative stress induced by ROS has been 
suggested to be a common mediator in apoptosis $(30,31)$ and particularly in DN, a state in which oxidative stress is increased by high glucose and dyslipidemia (32).

In conclusion, for the first time to the best of our knowledge, the present study provided evidence that ETF $\beta$ expression is decreased in kidneys of diabetics, and this is associated with TEC apoptosis and renal injury. Moreover, downregulation of ETF $\beta$ by siRNA induces apoptosis of renal tubular cells. In conclusion, decreased expression of ETF $\beta$ in DN is associated with TEC apoptosis during the progression of diabetes.

\section{Acknowledgements}

The present study was supported by a Project of International Collaboration in Science and Technology Grant, China (grant no. 2011DFA31860), the National Major Scientific and Technological Special Project for 'Significant New Drugs Development' during the Twelfth Five-year Plan Period (no. 2012ZX09103201-014) and the National Natural Science Foundation of China (grant no. 81173422 and 81130066). We gratefully acknowledge Frank J. Burczynski for a critical reading and language revision of the manuscript. We also thank Nissi S. Wang for assistance with editing the manuscript.

\section{References}

1. Shaheen FA and Al-Khader AA: Epidemiology and causes of end stage renal disease (ESRD). Saudi J Kidney Dis Transpl 6: 277-281, 2005

2. Okamura DM, Pasichnyk K, Lopez-Guisa JM, Collins S, Hsu DK, Liu FT and Eddy AA: Galectin-3 preserves renal tubules and modulates extracellular matrix remodeling in progressive fibrosis. Am J Physiol Renal Physiol 300: F245-F253, 2011.

3. Futrakul N and Futrakul P: Renal microvascular and tubular injuries in type II diabetic nephropathy. Kidney Int 74: 390 , author reply 390-391, 2008.

4. Beyenbach KW: Kidneys sans glomeruli. Am J Physiol Renal Physiol 286: F811-F827, 2004

5. Ishizaki K, Schauer N, Larson TR, Graham IA, Fernie AR and Leaver CJ: The mitochondrial electron transfer flavoprotein complex is essential for survival of Arabidopsis in extended darkness. Plant J 47: 751-760, 2006.

6. Covington MD and Schnellmann RG: Chronic high glucose downregulates mitochondrial calpain 10 and contributes to renal cell death and diabetes-induced renal injury. Kidney Int 81: 391-400, 2012.

7. Ruzicka FJ and Beinert H: A new iron-sulfur flavoprotein of the respiratory chain. A component of the fatty acid beta oxidation pathway. J Biol Chem 252: 8440-8445, 1977.

8. Toogood HS, Leys D and Scrutton NS: Dynamics driving function: new insights from electron transferring flavoproteins and partner complexes. FEBS J 274: 5481-5504, 2007.

9. Zhang J, Frerman FE and Kim JJ: Structure of electron transfer flavoprotein-ubiquinone oxidoreductase and electron transfer to the mitochondrial ubiquinone pool. Proc Natl Acad Sci USA 103: 16212-16217, 2006.

10. Goodman SI, Binard RJ, Woontner MR and Frerman FE: Glutaric acidemia type II: gene structure and mutations of the electron transfer flavoprotein:ubiquinone oxidoreductase (ETF:QO) gene. Mol Genet Metab 77: 86-90, 2002.

11. Kawano K, Hirashima T, Mori S, Saitoh Y, Kurosumi M and Natori T: Spontaneous long-term hyperglycemic rat with diabetic complications. Otsuka Long-Evans Tokushima Fatty (OLETF) strain. Diabetes 41: 1422-1428, 1992.

12. Zhao TT, Zhang HJ, Lu XG, Huang XR, Zhang WK, Wang $\mathrm{H}$, Lan HY and Li P: Chaihuang-Yishen granule inhibits diabetic kidney disease in rats through blocking TGF- $\beta / \mathrm{Smad} 3$ signaling. PLoS One 9: e90807, 2014.

13. Huang XR, Chung AC, Zhou L, Wang XJ and Lan HY: Latent TGF-betal protects against crescentic glomerulonephritis. J Am Soc Nephrol 19: 233-242, 2008.
14. Kelsen S, He X and Chade AR: Early superoxide scavenging accelerates renal microvascular rarefaction and damage in the stenotic kidney. Am J Physiol Renal Physiol 303: F576-F583, 2012.

15. Yang J, Liu X, Bhalla K, Kim CN, Ibrado AM, Cai J, Peng TI, Jones DP and Wang X: Prevention of apoptosis by Bcl-2: release of cytochrome $c$ from mitochondria blocked. Science 275: 1129-1132, 1997.

16. White KE and Bilous RW: Type 2 diabetic patients with nephropathy show structural-functional relationships that are similar to type 1 disease. J Am Soc Nephrol 11: 1667-1673, 2000.

17. Docherty NG, O'Sullivan OE, Healy DA, Fitzpatrick JM and Watson RW: Evidence that inhibition of tubular cell apoptosis protects against renal damage and development of fibrosis following ureteric obstruction. Am J Physiol Renal Physiol 290: F4-F13, 2006.

18. Kumar D, Robertson S and Burns KD: Evidence of apoptosis in human diabetic kidney. Mol Cell Biochem 259: 67-70, 2004.

19. Verzola D, Bertolotto MB, Villaggio B, Ottonello L, Dallegri F, Salvatore F, Berruti V, Gandolfo MT, Garibotto G and Deferrari G: Oxidative stress mediates apoptotic changes induced by hyperglycemia in human tubular kidney cells. J Am Soc Nephrol 15 (Suppl 1): S85-S87, 2004.

20. Chen JF, Liu H, Ni HF, Lv LL, Zhang MH, Zhang AH, Tang RN, Chen PS and Liu BC: Improved mitochondrial function underlies the protective effect of pirfenidone against tubulointerstitial fibrosis in 5/6 nephrectomized rats. PLoS One 8: e83593, 2013.

21. Lu H, Yang Y, Allister EM, Wijesekara N and Wheeler MB: The identification of potential factors associated with the development of type 2 diabetes: a quantitative proteomics approach. Mol Cell Proteomics 7: 1434-1451, 2008.

22. Yuan J and Horvitz HR: A first insight into the molecular mechanisms of apoptosis. Cell 116 (Suppl 2): S53-S56, 2004.

23. Huppertz B, Frank HG and Kaufmann P: The apoptosis cascade - morphological and immunohistochemical methods for its visualization. Anat Embryol (Berl) 200: 1-18, 1999.

24. Roberts DL, Frerman FE and Kim JJ: Three-dimensional structure of human electron transfer flavoprotein to 2.1-A resolution. Proc Natl Acad Sci USA 93: 14355-14360, 1996.

25. Wan J, Jiang L, Lü Q, Ke L, Li X and Tong N: Activation of PPARdelta up-regulates fatty acid oxidation and energy uncoupling genes of mitochondria and reduces palmitate-induced apoptosis in pancreatic beta-cells. Biochem Biophys Res Commun 391: 1567-1572, 2010

26. Qiu J, Gao HQ, Liang Y, Yu H and Zhou RH: Comparative proteomics analysis reveals role of heat shock protein 60 in digoxin-induced toxicity in human endothelial cells. Biochim Biophys Acta 1784: 1857-1864, 2008.

27. Bastin J, Djouadi F, Geloso JP and Merlet-Benichou C: Postnatal development of oxidative enzymes in various rat nephron segments: effect of weaning on different diets. Am J Physiol 259: F895-F901, 1990.

28. Hirokawa S, Shimanuki T, Kitajima H, Nishimori $\mathrm{Y}$ and Shimosaka M: Knockdown of electron transfer flavoprotein $\beta$ subunit reduced TGF- $\beta$-induced $\alpha$-SMA mRNA expression but not COL1A1 in fibroblast-populated three-dimensional collagen gel cultures. J Dermatol Sci 68: 179-186, 2012.

29. Rosca MG, Vazquez EJ, Chen Q, Kerner J, Kern TS and Hoppel CL: Oxidation of fatty acids is the source of increased mitochondrial reactive oxygen species production in kidney cortical tubules in early diabetes. Diabetes 61: 2074-2083, 2012.

30. Buttke TM and Sandstrom PA: Oxidative stress as a mediator of apoptosis. Immunol Today 15: 7-10, 1994.

31. Jee SH, Kim HJ and Lee J: Obesity, insulin resistance and cancer risk. Yonsei Med J 46: 449-455, 2005.

32. Horie K, Miyata T, Maeda K, Miyata S, Sugiyama S, Sakai H, van Ypersole de Strihou C, Monnier VM, Witztum JL and Kurokawa K: Immunohistochemical colocalization of glycoxidation products and lipid peroxidation products in diabetic renal glomerular lesions. Implication for glycoxidative stress in the pathogenesis of diabetic nephropathy. J Clin Invest 100: 2995-3004, 1997. 